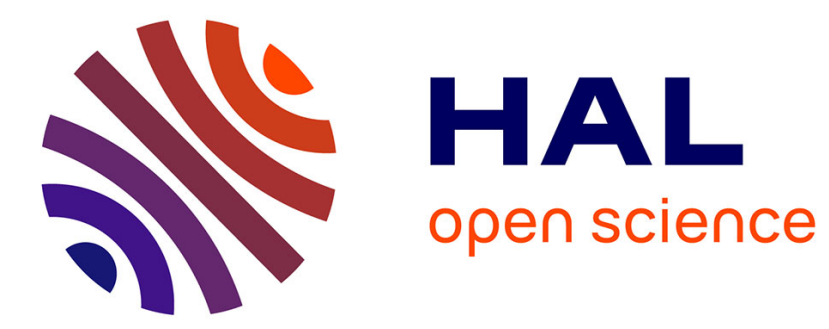

\title{
Qualité des produits biologiques d'origine animale
}

Marilyne Kouba

\section{To cite this version:}

Marilyne Kouba. Qualité des produits biologiques d'origine animale. Productions Animales, 2002, 15

(3), pp.161-169. hal-02681702

\section{HAL Id: hal-02681702 \\ https://hal.inrae.fr/hal-02681702}

Submitted on 31 May 2020

HAL is a multi-disciplinary open access archive for the deposit and dissemination of scientific research documents, whether they are published or not. The documents may come from teaching and research institutions in France or abroad, or from public or private research centers.
L'archive ouverte pluridisciplinaire HAL, est destinée au dépôt et à la diffusion de documents scientifiques de niveau recherche, publiés ou non, émanant des établissements d'enseignement et de recherche français ou étrangers, des laboratoires publics ou privés. 
INRA Prod. Anim., 2002, 15 (3), 161-169

\section{KOUBA}

INRA, UMRVP, 35590 Saint-Gilles

Courriel :

kouba@st-gilles.rennes.inra.fr

\section{Qualité des produits biologiques d'origine animale}

Les aliments bio sont-ils meilleurs pour notre santé ? Difficile de répondre à la question puisqu'il n'y a pas d'étude épidémiologique sur la relation entre consommation de produits biologiques et santé humaine. Le but de cet article n'est pas de polémiquer sur ce sujet, mais de présenter les études scientifiques comparant les productions animales conventionnelle et biologique.

Les récentes crises européennes relatives à l'élevage (ESB, dioxine, fièvre aphteuse) ont effrayé les consommateurs qui se tournent de plus en plus vers les produits biologiques. De nombreuses études et enquêtes réalisées en Europe ont en effet révélé que le choix des consommateurs est motivé essentiellement par la salubrité et l'innocuité des aliments biologiques, bien que les préoccupations écologiques et le souci du bien-être des animaux jouent également un rôle primordial (Sylvander 1999). Ainsi, une étude menée en

\section{Résumé}

L'appellation biologique pour un produit garantit un mode de production selon la réglementation spécifique à l'agriculture biologique. Il existe en effet un Règlement Européen pour les Productions Animales Biologiques, appelé REPAB, dont l'application dans le droit français (en date du 24 août 2000) a fait l'objet de contraintes supplémentaires : c'est le CC REPAB F. Il existe une forte demande en produits biologiques dans les pays industrialisés. Il est par conséquent important de considérer à la fois les qualités nutritionnelle, organoleptique et hygiénique de ces produits. Cependant, une réelle comparaison avec des produits conventionnels est difficile car, par exemple, les techniques d'élevage biologique peuvent être différentes entre elles, et notamment utiliser des races différentes. Il existe peu de données publiées exploitables, et il n'y a pas de résultat montrant une réelle différence entre les produits animaux biologiques et les produits animaux conventionnels, en termes de qualités organoleptique et nutritionnelle. Les produits biologiques ont de plus faibles teneurs en résidus de substances vétérinaires médicamenteuses et de pesticides. L'appellation biologique donne également aux consommateurs l'assurance que les aliments n'ont pas été soumis à l'ionisation et l'utilisation des OGM est bannie dans le cadre de la réglementation de l'agriculture biologique. Mais l'agriculture biologique conduirait à un risque accru de contamination des produits d'origine animale par les microorganismes présents chez les animaux et dans leurs déjections. En résumé, selon les critères, les produits biologiques ont de moins bons ou de meilleurs résultats que les produits conventionnels.
1997 au Royaume-Uni a montré que le premier critère d'achat pour $46 \%$ des consommateurs de produits biologiques est la sécurité alimentaire (IFST 2001). Selon une étude réalisée en France en février 2001, pour $61 \%$ des Français le logo AB (Agriculture Biologique) est le signe d'une absence totale de substances chimiques exogènes dans le produit destiné à la consommation humaine (INC 2001).

De 29000 en 1993, le nombre d'exploitations pratiquant l'agriculture biologique ou en conversion est passé à 120000 en 1999 pour les 15 pays de l'Union Economique Européenne, ce qui correspond à $1,7 \%$ des exploitations et 2,6\% de la surface agricole utile (Europa 2001).

La véritable question que l'on peut se poser est la suivante : la nourriture biologique estelle meilleure pour nous ? Etant donné l'absence d'étude épidémiologique sur la relation entre la consommation de produits biologiques et la santé humaine, il est très difficile de répondre à la question. Il existe une véritable polémique à ce sujet, certains n'hésitant pas à prêter des vertus thérapeutiques aux produits biologiques, notamment dans le cas de cancers (Bishop 1988), bien que ces affirmations n'aient jamais été prouvées d'un point de vue scientifique (Saffron 1999). Le but de cet article n'est pas de prendre parti, mais de présenter les études scientifiques comparant les productions animales conventionnelle et biologique. Cet objectif peut sembler très simple ; il est en fait difficile à 
atteindre car il existe peu d'études comparatives, celles-ci portant surtout sur les bovins, alors que les études sur le porc et la volaille sont rares.

L'agriculture biologique est strictement réglementée : les ministres européens de l'agriculture ont adopté le 19 juillet 1999 le règlement $\mathrm{CE} \mathrm{n}^{\circ}$ 1804/99 (Règlement Européen pour les Productions Animales Biologiques appelé $\mathrm{REPAB})$. Ce règlement est entré en application en France par un arrêté ministériel en date du 24 août 2000. La France a élaboré un cahier des charges complétant ce règlement : le CC REPAB F. Ce texte décrit en particulier le mode de production biologique (alimentation des animaux, soins et prophylaxie ...) et présente une liste de fertilisants et produits de traitements, matières premières, additifs, pouvant être utilisés en agriculture biologique. Cependant, de nombreux produits synthétiques sont interdits : c'est le cas de pesticides, herbicides, fongicides .... L'utilisation des traitements allopathiques curatifs ou des antibiotiques est très limitée et sous la responsabilité d'un vétérinaire. L'incorporation d'antibiotiques et autres additifs aux aliments des animaux ainsi que l'utilisation des hormones et des promoteurs de croissance sont prohibées.

Par conséquent, l'existence de résidus de ces substances dans les produits biologiques est hautement improbable. Ceci est à la base même $\mathrm{du}$ fait que les consommateurs considèrent que les produits biologiques sont plus sains que les produits conventionnels (INC 2001).

Dans les pays de l'Union européenne est interdite sur le produit biologique toute indication suggérant au consommateur une supériorité sensorielle, nutritionnelle ou sanitaire. Cependant, de telles indications ne sont pas nécessaires quand pour le consommateur l'intitulé biologique est synonyme de qualité supérieure (rapport FAO 2000)

Les aliments biologiques d'origine animale sont produits de diverses façons, parfois à partir de races animales particulières, ce qui rend difficile la comparaison avec une production conventionnelle. D'après le nombre limité de résultats disponibles, il apparaît que la qualité des produits, tant nutritionnelle

Tableau 1. Différence (en pourcentage) de composition du lait biologique en comparaison au lait conventionnel.

\begin{tabular}{|l|c|c|}
\hline & Guinot-Thomas et al 1991 & Lund 1991 \\
\hline Matière sèche & $-0,2$ & $+4,5$ \\
Lipides & $-3,5$ & $+7,0$ \\
Protéines & - & $+7,5$ \\
Calcium & $+4,9$ & $+3,5$ \\
\hline
\end{tabular}

qu'hygiénique, n'est pas très différente dans les deux types de production (Honikel 1998). C'est cette qualité que nous allons examiner dans cette étude.

\section{1 / Qualités nutritionnelles et organoleptiques}

Les études comparatives sur les végétaux, plus nombreuses que celles effectuées sur animaux, indiquent que les produits biologiques contiendraient plus de nutriments que les produits issus de l'agriculture conventionnelle (Hornick 1992, Smith 1993). Ce phénomène pourrait être dû à la teneur en eau plus élevée des végétaux conventionnels, qui conduirait à une dilution des nutriments (Lecerf 1995, Worthington 1998).

Plusieurs études ont comparé la qualité du lait issu de l'élevage conventionnel et biologique (Gedek et al 1981, Arnold 1984, Knöppler et Averdunk 1986, Gravert et al 1989, Guinot-Thomas et al 1991, Lund 1991). Dans ces travaux, les deux formes de production diffèrent surtout par la nature de l'aliment distribué aux animaux. Ces études ne mettent pas en évidence d'importantes différences entre les deux types de production, en ce qui concerne la composition du lait (Guinot-Thomas et al 1991, Lund 1991 ; tableau 1).

Des poules identiques génétiquement, mais élevées selon des conditions différentes produisent des oufs de caractéristiques différentes (tableau 2). Les oufs de poules ayant reçu une alimentation biologique ont une teneur protéique plus faible et une teneur en lécithine plus élevée que les œufs de poules ayant reçu un aliment conventionnel (Plochberger 1989). On observe généralement une plus forte teneur en caroténoïdes des œufs produits par des poules élevées au sol avec accès à un parcours extérieur, comparées aux poules en cage ou au sol mais sans parcours extérieur, qui s'explique par la consommation d'herbe et de plantes vertes sur le parcours extérieur par les premières, ce qui constitue un apport supplémentaire en caroténoïdes (Woese et al 1997).

Sundrum et al (2000) ont observé que la suppression des acides aminés de synthèse dans l'alimentation du porc, comme c'est le cas en agriculture biologique, a pour conséquence une augmentation de la teneur en tissu adipeux intramusculaire (tableau 3), ce qui est favorable en terme de qualité organoleptique. De même, Woodward et Fernandez (1999) ont montré que la viande de boufs en finition biologique présente plus de persillé que celle de boufs en finition conventionnelle. Cependant, Hansson et al (2000) ont quant

Tableau 2. Poids des œufs et composition en albumen, vitellus et coquille (Plochberger 1989).

\begin{tabular}{|l|c|c|c|}
\hline & Poules 'biologiques' & Poules 'standard' & Ecart significatif \\
\hline Poids des oeufs (g) & 55,1 & 51,9 & $\mathrm{P}<0,01$ \\
\hline Albumen (\%) & 54,6 & 55,7 & $\mathrm{P}<0,05$ \\
\hline Vitellus (\%) & 35,0 & 33,8 & $\mathrm{P}<0,05$ \\
\hline Coquille (\%) & 10,4 & 10,4 & non \\
\hline
\end{tabular}


Tableau 3. Caractéristiques des carcasses de porc selon le régime alimentaire (Sundrum et al 2000).

\begin{tabular}{|l|c|c|}
\hline \multicolumn{1}{|c|}{ Aliment } & conventionnel & biologique $^{(1)}$ \\
\hline Poids à l'abattage (kg) & 93,1 & 91,2 \\
Rendement de carcasse (\%) & 77,9 & 76,7 \\
Viande maigre (fat'o-meter) (\%) & $56,0^{\mathrm{a}}$ & $54,3^{\mathrm{b}}$ \\
Epaisseur de bardière (cm) & 2,4 & 2,4 \\
Gras intramusculaire (\%) & $1,2^{\mathrm{a}}$ & $2,9^{\mathrm{b}}$ \\
\hline
\end{tabular}

Sur une ligne, les valeurs suivies de lettres différentes diffèrent significativement à au moins $P<0,05$.

(1) Régime à base de pois et lupin, sans supplémentation en acides aminés.

Figure 1. Etat d'engraissement de jeunes taureaux, en fonction de leur mode d'élevage, biologique ou conventionnel (Hansson et al 2000).

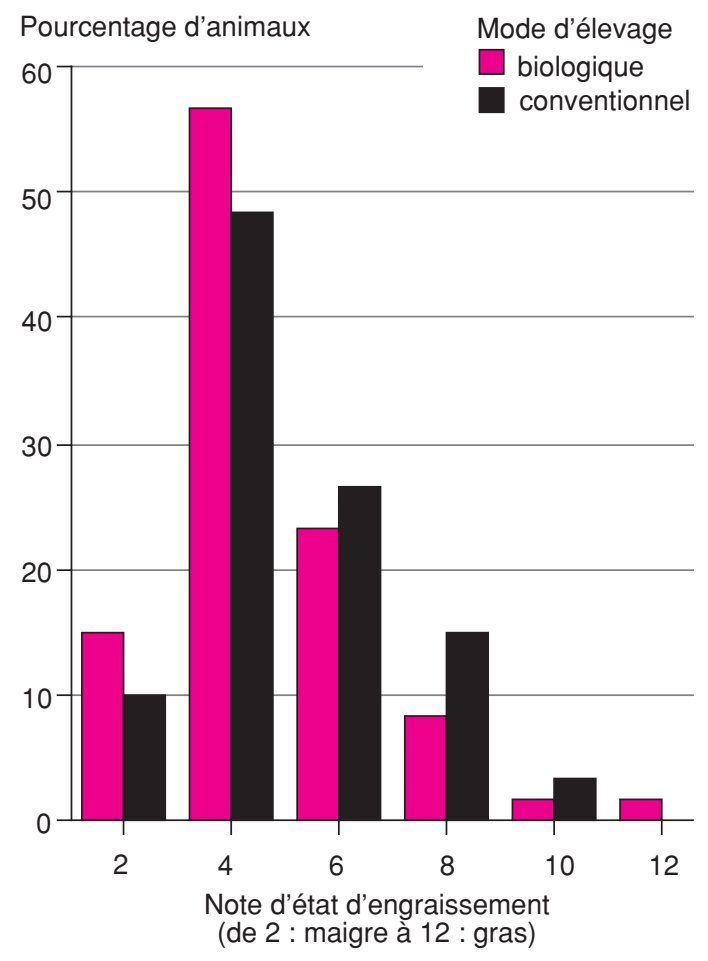

à eux mis en évidence que la carcasse de bovins élevés biologiquement était plus maigre que celle de bovins conventionnels (figure 1). Les résultats de ces études portant sur l'engraissement des animaux biologiques sont donc contradictoires.

Les produits biologiques présentent-ils donc une supériorité organoleptique ? Certains le prétendent, mais il n'a jamais été mis en évidence de différence de qualité organoleptique entre les produits biologiques et conventionnels, car peu voire pas d'études comparatives ont été réalisées.

\section{2 / Qualité hygiénique : résidus chimiques}

En production biologique, l'utilisation des pesticides est interdite. Par conséquent, les produits biologiques devraient contenir moins de résidus de pesticides que les produits issus de l'agriculture conventionnelle. De manière générale, en terme de santé humaine, les risques dus aux additifs alimentaires et aux résidus de pesticides sont relati- vement faibles, comparés aux risques microbiologiques et aux risques dus aux toxines naturelles. Une étude épidémiologique réalisée en France sur les toxi-infections alimentaires a montré que seulement $0,5 \%$ des cas étaient dus à une contamination par une substance chimique. Cependant, en Europe, la contamination des aliments par des produits chimiques est perçue comme un important problème de santé publique.

\section{1 / Substances médicamen- teuses dans les aliments des animaux}

Selon la réglementation européenne, l'utilisation des substances médicamenteuses est très limitée et contrôlée dans les productions animales biologiques. La gestion de la santé des animaux est basée sur la prévention des pathologies par des compléments alimentaires comme des oligo-éléments ou des algues, par des mesures de prophylaxie, par l'utilisation de l'homéopathie, de la phytothérapie ... et par des mesures comme la sélection d'animaux résistants, un régime alimentaire équilibré et de très bonne qualité ainsi qu'un environnement favorable.

Les problèmes de santé publique engendrés par la résistance de plus en plus forte des microorganismes aux antibiotiques, ainsi que la forte médiatisation de l'utilisation de l'hormone de croissance bovine aux USA, ont engendré une demande en produits laitiers biologiques qui augmente de $35 \%$ par an aux Etats-Unis (Sloan 1998). Cependant, certains des microorganismes pathogènes que l'utilisation des antibiotiques à titre préventif contrôle, représentent des menaces potentielles pour la santé humaine. Une ancienne étude montre également que lorsqu'il y a résidus de substances médicamenteuses (production conventionnelle), ceux-ci sont toujours présents en quantité extrêmement faible et ne présentent pas de menace pour la santé du consommateur (Jukes 1977). On peut cependant s'interroger sur le fait que depuis cette époque, la teneur en substances médicamenteuses des produits alimentaires d'origine animale a peut-être évolué.

\section{2 / Les contaminants dans l'alimentation des animaux}

Les contaminants des aliments destinés aux animaux, comme les résidus de pesticides, les produits chimiques et industriels et les métaux lourds, peuvent se retrouver dans la 


\section{Le risque de contamination par des résidus \\ chimiques est réduit dans les produits bio, mais il existe, du fait de la présence de polluants dans l'environnement.}

viande, le lait ou les œufs issus de ces animaux. Comme la réglementation européenne impose que les animaux élevés selon le mode biologique reçoivent une alimentation biologique, le risque de contamination éventuelle par des résidus de pesticides et d'autres substances chimiques est réduit, en comparaison avec le mode d'élevage conventionnel. Cependant, les produits issus de l'agriculture biologique peuvent être contaminés par des polluants présents dans l'environnement immédiat de l'exploitation agricole. Ces polluants qui peuvent contaminer les aliments des animaux pourront éventuellement se retrouver dans les produits issus de ces animaux et destinés à la consommation humaine. En 1998, une étude menée en France par l'Institut National de la Consommation a montré que les teneurs en dioxine étaient similaires dans des laits biologiques et conventionnels (avec des teneurs comprises entre 0,34 et 0,73 picogrammes par gramme de matière grasse). Ces valeurs sont cependant plus faibles que la valeur maximale autorisée par l'Union européenne (1 picogramme par gramme de matière grasse) (INC 1998). Dans un article de synthèse allemand portant sur plus de 100 études, les auteurs concluent que la teneur en pesticide de céréales, pommes de terre, légumes, fruits, vin, pain et lait est similaire en production biologique et conventionnelle (Woese et al 1997). Cependant, la faible qualité de beaucoup de ces études les rend peu fiables et rend toute généralisation difficile. D’autres études menées sur la présence

Figure 2. Présence de résidus de DDT (dichloro diphényl trichloroéthane, exprimés en ppb) dans des laits biologiques et conventionnels (Maruejouls et Goulard 1999).

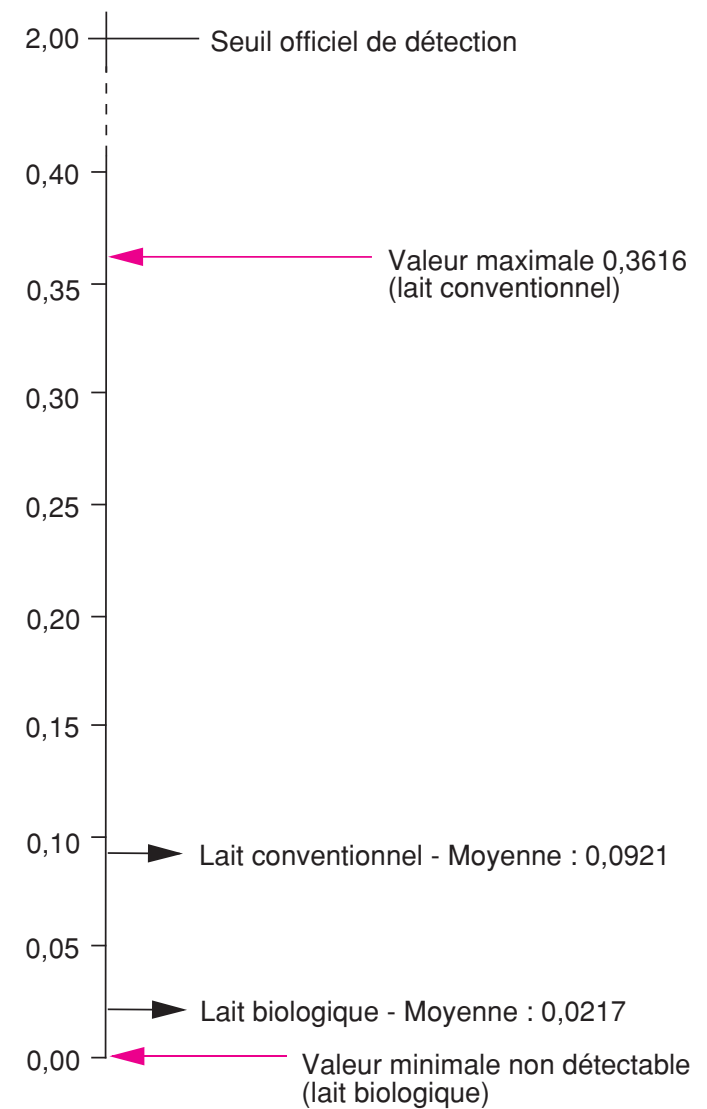

éventuelle de résidus de pesticides dans des produits biologiques et conventionnels mettent en évidence une présence plus faible de ces résidus dans les produits biologiques, bien que ces produits ne puissent pas être définis comme étant indemnes de pesticides (Lecerf 1995, Maruejouls et Goulard 1999 ; figures 2 et 3 ).

Certaines études ont montré que la concentration spermatique était plus élevée chez des agriculteurs et des consommateurs ayant opté pour le mode biologique comparés à d'autres hommes (Abell et al 1994, Jensen et al 1996), mais des études plus récentes n'ont pas confirmé ces résultats (Juhler et al 1999, Larsen et al 1999 ; tableau 4). En effet, Ratcliffe et al (1987) ont mis en évidence qu'une exposition à des quantités élevées de pesticides peut augmenter le risque de

Tableau 4. Concentration spermatique (millions de $s p z / m l)$ dans une population de consommateurs et de producteurs «biologiques» comparés à une population de consommateurs et de producteurs «conventionnels».

\begin{tabular}{|l|c|c|}
\hline Population & «biologique» & «conventionnelle» \\
\hline Abell et al 1994 & $100^{\text {a }}$ & $50^{\mathrm{b}}$ \\
\hline Jensen et al 1996 & $69^{\mathrm{a}}$ & $48^{\mathrm{b}}$ \\
\hline Juhler et al 1999 & 75 & 62 \\
\hline Larsen et al 1999 & 64 & 58 \\
\hline
\end{tabular}

Sur une ligne, les valeurs suivies de lettres différentes diffèrent significativement à $\mathrm{P}<0,01$.

Figure 3. Présence de résidus de Lindane (exprimés en ppb) dans des laits biologiques et conventionnels (Maruejouls et Goulard 1999).

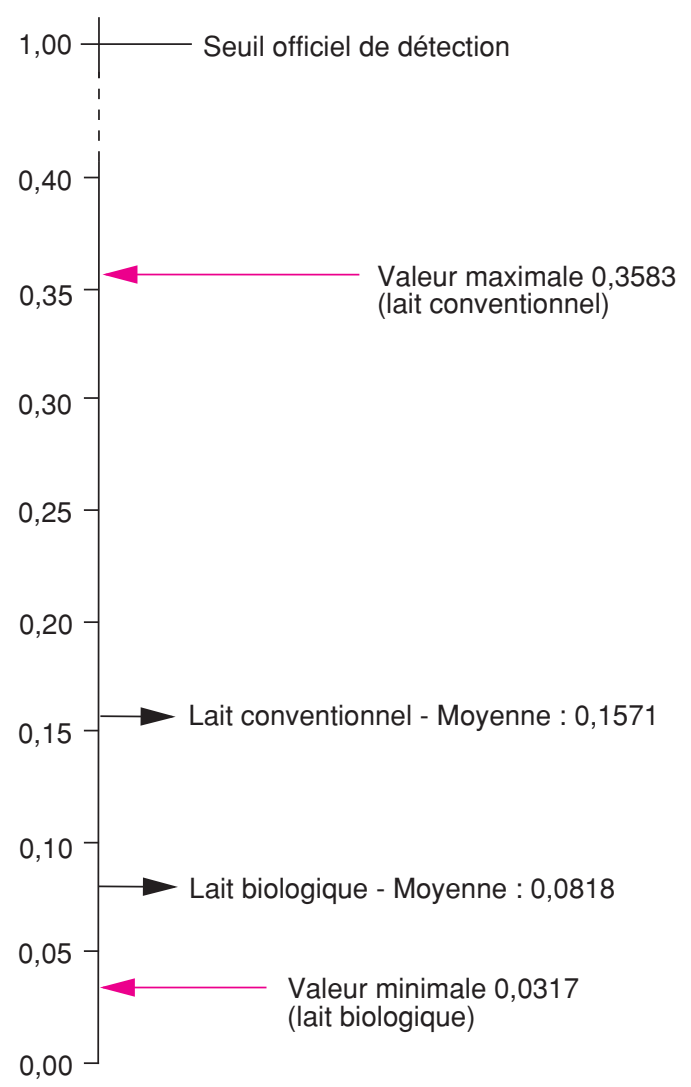


Tableau 5. Concentration spermatique (millions de $s p z / m l$ ) et qualité des spermatozoïdes dans une population d'ouvriers exposés à des teneurs élevées en pesticide (dibromide éthylène) comparée à une population non exposée (Ratcliffe et al 1987).

\begin{tabular}{|l|c|c|c|}
\hline Population & non exposée & exposée & Ecart significatif \\
\hline Concentration & 59,4 & 48,4 & non \\
\hline Viabilité (\%) & 85,5 & 68,1 & $\mathrm{P}<0,01$ \\
\hline Motilité (\%) & 46,6 & 35,2 & $\mathrm{P}<0,05$ \\
\hline Formes normales (\%) & 82,7 & 78,9 & $\mathrm{non}$ \\
\hline Absence de tête (\%) & 1,4 & 2,1 & $\mathrm{P}<0,001$ \\
\hline Queues anormales (\%) & 5,7 & 6,5 & $\mathrm{P}<0,001$ \\
\hline
\end{tabular}

troubles de la reproduction masculine (tableau 5). Une étude plus récente (Oliva et al 2001) montre également que l'exposition à des pesticides et à des solvants est, de façon significative, positivement associée à des problèmes de stérilité masculine.

La peur des résidus de pesticides dans l'alimentation a été utilisée pour promouvoir la vente de produits biologiques. Cependant, lorsque l'on compare la très faible quantité de résidus de pesticides auxquels nous sommes exposés à travers l'alimentation, conventionnelle comme biologique, à l'exposition massive aux pesticides synthétiques et naturels provenant d'autres sources, il est hautement improbable que les résidus de pesticides dans les aliments soient une cause significative de pathologies chez les consommateurs.

\section{3 / Qualité hygiénique : contaminations micro- biennes et parasitaires}

\section{1 / Contamination par les engrais naturels}

L'agriculture biologique utilise comme engrais essentiellement les déjections animales. Ces fertilisants naturels sont également largement utilisés en agriculture conventionnelle en même temps que les engrais synthétiques minéraux. Le risque de contamination microbiologique résultant de l'utilisation de ces engrais naturels et les méthodes à mettre en ouvre pour réduire ce risque sont donc à envisager dans les deux types d'agriculture.

Les déjections animales non traitées ou mal traitées utilisées comme engrais peuvent être une source de contamination des produits et/ou de l'eau du sol. Elles contiennent en effet de nombreux agents pathogènes pour l'homme et doivent subir un traitement adapté avant utilisation.

La plupart des recherches sur le compostage et l'épandage ont surtout examiné les effets de différentes pratiques sur la fertilité $\mathrm{du}$ sol et la qualité des récoltes. Des recherches sont encore à mener sur la survie des agents pathogènes dans les effluents d'élevage non traités, leur mode de dissémination dans les champs, ainsi que sur les traitements à mettre en ouvre pour diminuer leurs teneurs ou les éliminer complètement.
Une étude menée en 1995 indique que certains microorganismes peuvent survivre pendant 59 jours dans des conditions de compostage (Droffner et Brinton 1995). Certains agents pathogènes, comme le virus de l'hépatite $\mathrm{A}$, ont une résistance thermique beaucoup plus élevée que d'autres microorganismes.

\section{2 / Contamination bactérienne}

Selon l'organisme américain Centre for Disease Control (CDC), il y a aux Etats-Unis un total d'au moins 76 millions de cas d'intoxication alimentaire par an. Selon le CDC, la principale source d'infection humaine par $E$. coli est la viande contaminée durant l'abattage des animaux. Des recherches menées à l'Université de Cornell ont montré que des souches virulentes d'E. coli, telles que $E$. coli 0157 : H7 se développent dans l'appareil digestif des bovins lorsque ceux-ci sont surtout nourris avec des aliments riches en amidon. Lorsque les animaux sont nourris avec du foin, on retrouve dans leurs fèces moins de $1 \%$ de la quantité d'E. coli présente dans les fèces des bovins nourris au grain (Couzin 1998). Lalimentation des ruminants élevés selon le mode biologique étant basée sur des fourrages, le risque d'infection de ces animaux par E. coli apparaît donc plus faible. En ce qui concerne la qualité du lait, une étude rapportée par Sundrum (2001) n'a pas mis en évidence de différence de contamination microbienne entre lait biologique et lait conventionnel. Cependant, selon un rapport édité par l'Union européenne en 2001, comparé à l'agriculture conventionnelle, le mode de production biologique est responsable d'un plus grand nombre de salmonelles dans les oufs, la volaille et la viande de porc (Europa 2001).

\section{3 / Mycotoxines}

Les mycotoxines sont des toxines produites par certaines moisissures qui peuvent se développer sur les aliments. Les aflatoxines sont les plus toxiques et peuvent induire un cancer du foie par ingestion de petites quantités de nourriture contaminée pendant une longue période de temps. Comme l'utilisation des fongicides est interdite en production biologique et comme les mycotoxines sont particulièrement dangereuses pour les animaux et pour l'homme, plusieurs études ont porté sur leur présence relative en production biologique et conventionnelle (Marx et al 1995, Skaug 1999). On ne peut pas conclure de ces

\section{L'élevage bio ne semble pas augmenter le risque de contamination microbienne du lait, mais il serait à l'origine d'une augmentation des salmonelles chez les porcs et les volailles.}


études que la production biologique puisse conduire à une augmentation du risque de contamination par les mycotoxines. De même, l'étude de la littérature spécialisée effectuée en l'an 2000 par la FAO n'a pas permis de confirmer une contamination accrue en agriculture biologique (rapport FAO 2000). Il est important de souligner que dans les deux systèmes de production, biologique et conventionnel, de bonnes pratiques culturales et de stockage permettent de minimiser le risque de développement de moisissure et de contamination par des mycotoxines. Deux études rapportées par Woese et al (1997) mettent en évidence un niveau d'aflatoxine M1 inférieur dans du lait biologique comparé à du lait conventionnel. Skaug (1999) a trouvé le même résultat pour le niveau de contamination par l'ochratoxine A du lait norvégien.

\section{4 / Parasites}

L'utilisation en routine des anti-parasitaires est interdite en production biologique et le nombre de traitements anti-parasitaires autorisés par le CC REPAB F est très limité et variable en fonction des espèces et des stades physiologiques. La proportion d'élevages avec parcours est plus élevée dans cette produc- tion. Par conséquent, on peut suspecter un plus grand risque de contamination des produits par des parasites en élevage biologique. Des études menées en Suède ont montré que les éleveurs du système biologique font état de la présence de parasites (aussi bien internes qu'externes) plus souvent que ne le font les éleveurs du système conventionnel (Dimander et al 2000, Svensson et al 2000 ; tableau 6). Ceci peut être dû, soit à un traitement inefficace, soit à une observation plus attentive des animaux dans le système biologique. Plusieurs études menées dans des pays du Nord au climat tempéré ont montré que la production en plein air de porcs ainsi que celle de poules pondeuses a pour conséquence une plus forte prévalence de parasites internes, lorsque l'on compare ce système plein air au système d'élevage conventionnel en bâtiment (Permin et al 1999, Thamsborg et al 1999 ; figures 4 et 5). Ceci indique donc que l'élevage biologique sans utilisation d'antiparasitaire a pour conséquence un parasitisme accru des animaux. Mais, dans la majorité des cas, ces parasites ne sont pas transmis à l'homme car ils sont soit éliminés à l'abattage avec le tube digestif, soit éliminés lors de la cuisson.

Tableau 6. Proportion d'éleveurs suédois rapportant des troubles dus à une helminthose chez des veaux laitiers (Svensson et al 2000).

\begin{tabular}{|l|c|c|c|}
\hline Elevages & biologiques & conventionnels & Ecart significatif \\
\hline Plus faible gain de poids & $17 \%$ & $5 \%$ & $\mathrm{P}<0,01$ \\
\hline Diarrhée & $14 \%$ & $6 \%$ & $\mathrm{P}<0,05$ \\
\hline Toux & $2 \%$ & $2 \%$ & non \\
\hline Lésions dans la viande & $11 \%$ & $7 \%$ & non \\
\hline Parasitisme pulmonaire & $5 \%$ & $4 \%$ & non \\
\hline Douve du foie & $35 \%$ & $27 \%$ & non \\
\hline
\end{tabular}

Figure 4. Contamination par Ascaris chez les porcs de différents stades physiologiques et dans différents systèmes de production (revue de Thamsborg et al 1999).

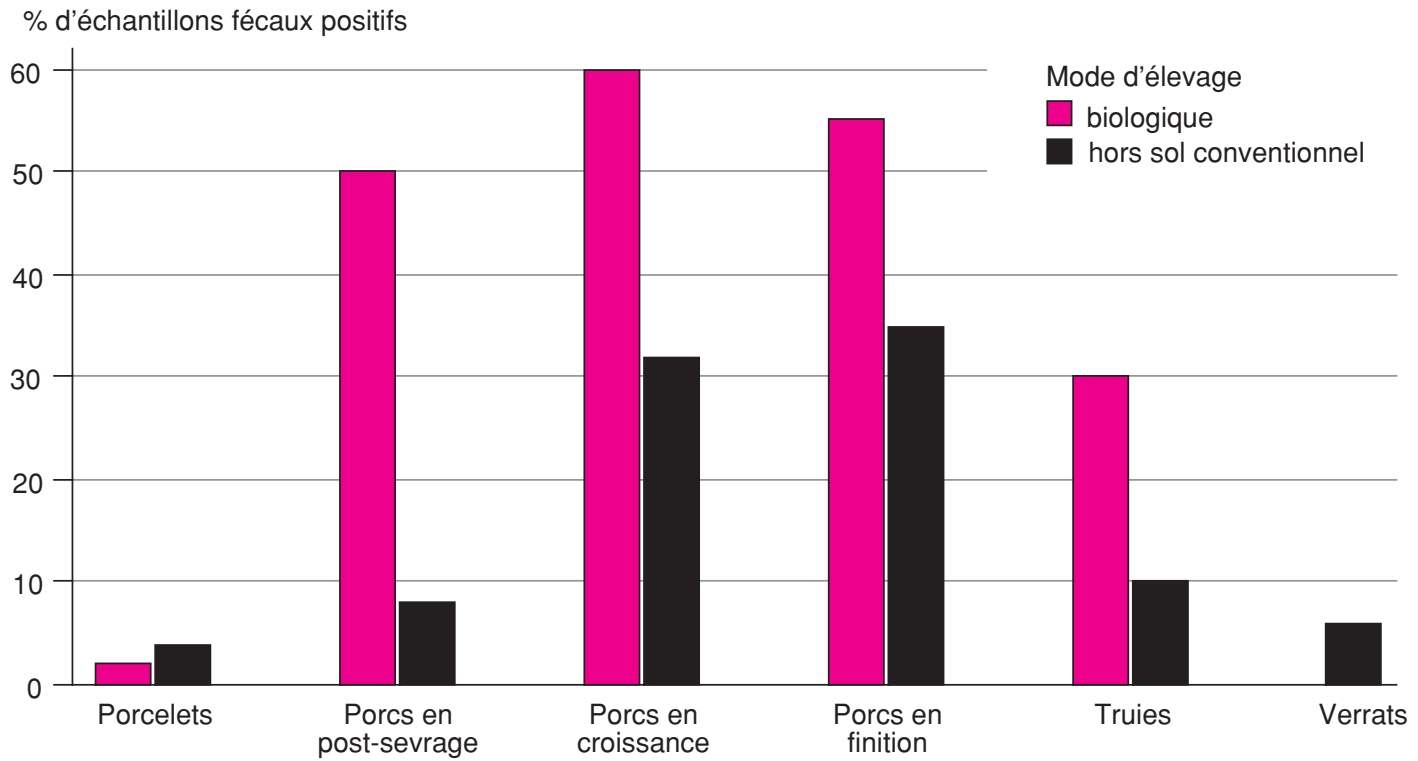


Figure 5. Prévalence d'helminthoses gastrointestinales chez des poules danoises (Permin et al 1999).

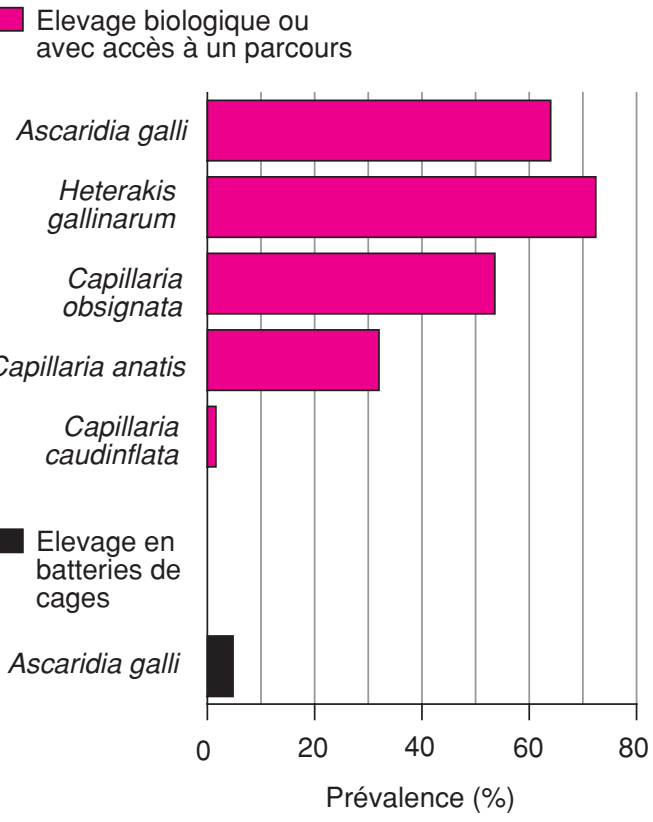

\section{4 / Autres critères de qualité}

\section{1 / Ionisation}

L'irradiation des denrées alimentaires permet le contrôle des insectes, parasites, bactéries pathogènes et des modifications qui peuvent intervenir en cours de conservation. Un rapport émis en 1997 par un comité d'experts mixte Food and Agriculture Organization of the United Nations (FAO) / International Atomic Energy Agency (IAEA) / World Health Organization (WHO) conclut « qu'une dose apte à atteindre l'objectif technologique recherché est à la fois sans danger pour le consommateur et adéquat d'un point de vue nutritionnel » (Who 1997). Cependant, ce procédé technologique n'est pas encore totalement accepté par tous les consommateurs. Ces derniers sont rassurés par le fait que les denrées alimentaires biologiques ne sont pas sujettes à des radiations ionisantes.

\section{2 / Les organismes génétique- ment modifiés (OGM)}

La réglementation européenne de la production biologique bannit l'utilisation des OGM, car les manipulations génétiques sont considérées comme étant incompatibles avec les principes mêmes de la production biologique. Lopinion publique redoute de possibles effets négatifs de cette technique sur l'environnement comme sur la santé humaine et animale, même s'il n'y a pas de preuve scientifique allant dans ce sens. Le choix du « biologique » est un moyen pour le consommateur d'éviter les OGM dans son alimentation.

\section{3 / La préférence alimentaire des animaux pour les aliments biologiques}

Deux études ont montré que les animaux consomment de plus grandes quantités d'aliment biologique, lorsqu'ils ont le choix, quel que soit leur précédent régime alimentaire (Plochberger 1989, Plochberger et Velimirov 1992 ; figures 6 et 7). Ceci n'est pas la preuve que la nourriture biologique est plus saine que la nourriture conventionnelle, mais c'est une observation intéressante, qui pourrait éventuellement trouver une application dans l'utilisation de ces animaux pour la réalisation de tests alimentaires.

\section{Conclusion}

L'agriculture biologique interdit la majorité des produits chimiques de traitement du sol

Figure 6. Quantité journalière de betteraves biologiques et conventionnelles consommées par des rats durant le test de préférence (Plochberger et Velmirov 1992).

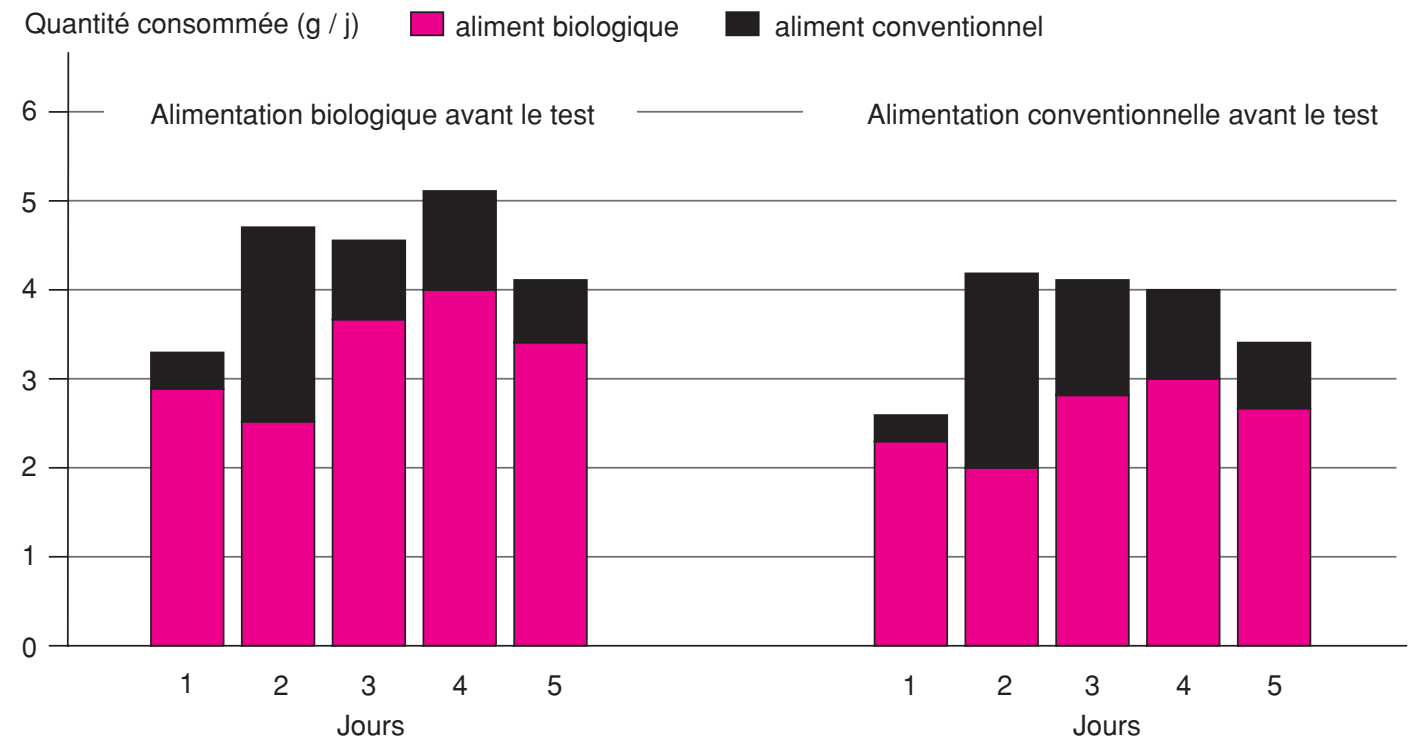


Figure 7. Quantité totale de betteraves biologiques et conventionnelles consommées par des poules durant le test de préférence (Plochberger 1989).

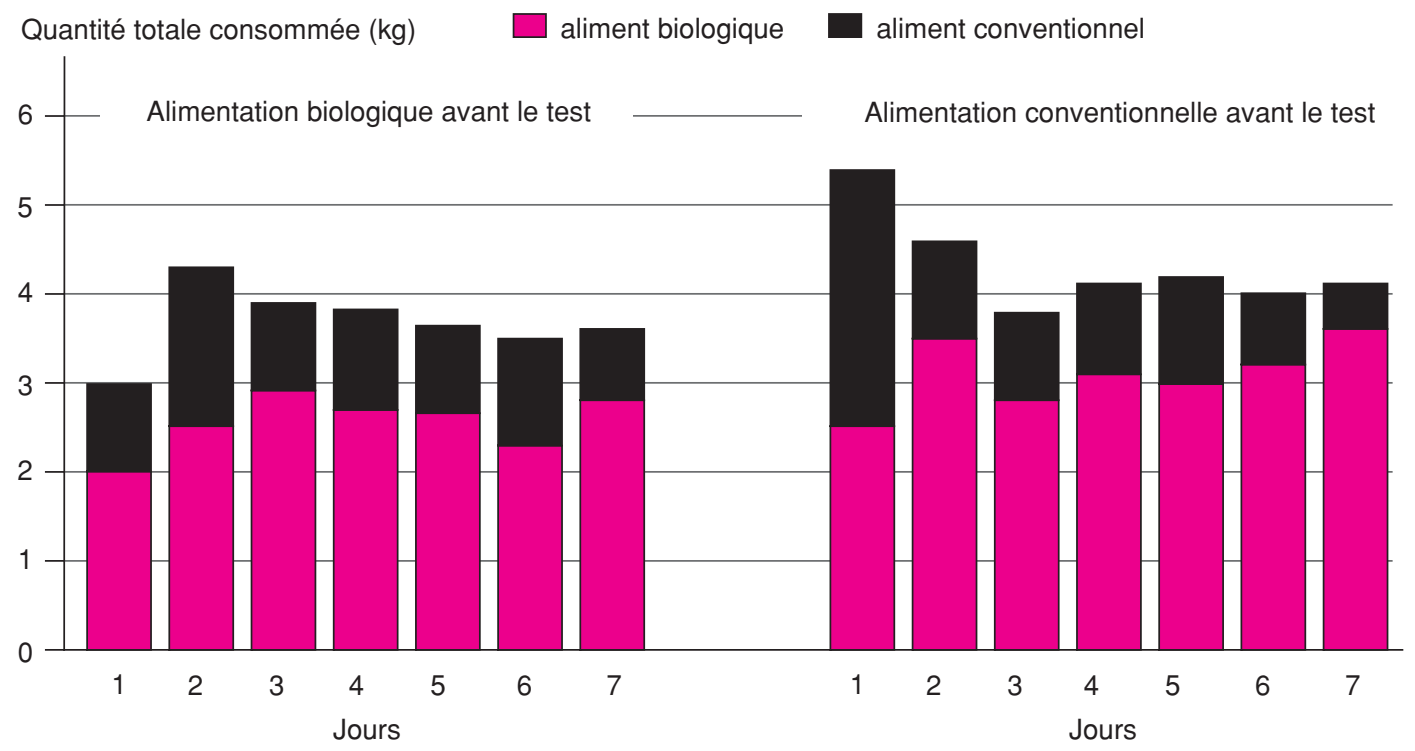

ou des animaux. Un certain nombre d'études ont été menées sur la qualité et l'hygiène des produits issus de ce type d'agriculture. Il semble que les produits animaux biologiques contiennent moins de résidus de pesticides et de produits médicamenteux. Enfin, les produits biologiques ne peuvent être soumis à l'ionisation et l'utilisation des OGM est exclue dans le système de production biologique. Cependant, ce dernier conduit à une plus forte contamination des animaux d'élevage par les parasites, internes et externes.
Il y a une demande croissante en produits biologiques de la part du consommateur qui perçoit l'agriculture biologique comme étant respectueuse de l'environnement et donnant des produits de meilleure qualité. Il est vraisemblable que la demande continuera d'augmenter dans le futur. Il est donc nécessaire de mettre en place des études épidémiologiques sur les implications en terme de santé humaine d'une consommation accrue de produits alimentaires biologiques.

\section{Références}

Abell A., Ernst E., Bonde J.P., 1994. High sperm density among members of organic farmers' association. The Lancet, 343, 1498.

Arnold Von R., 1984. Vergleichende qualitätsuntersuchungen von konventionell und alternativ erzeugter konsummilch. Arch. Lebensmittelhyg., 35, 66-69.

Bishop B., 1988. Organic food in cancer therapy. Nutr. Health, 6, 105-109.

Couzin J., 1998. Cattle diet linked to bacterial growth. Science, 281, 1578-1579.

Dimander S.O., Höglund J., Spörndly E., Waller P.J., 2000. The impact of internal parasites on the productivity of young cattle organically reared on semi-natural pastures in Sweden. Vet. Parasitol., 90, 271-284.

Droffner M.L., Brinton W.F., 1995. Survival of E. coli and Salmonella populations in aerobic thermophilic composts as measured with DNA gene probes. Zbl. Hyg., 197, 387-397.

Europa, 2001. Lagriculture biologique dans l'UE : faits et chiffres. http://europa.eu.int/

FAO, 2000. Food safety and quality as affected by organic farming. $22^{\text {nd }} \mathrm{FAO}$ regional conference for Europe, Porto, Portugal, 24-28 July 2000 (report).

Gedek von W., Knöppler H.O., Averdunk G., 1981. Vergleichende qualitätsuntersuchungen von milch aus landwirtschaftlichen betrieben mit konventioneller und mit alternativ wirtschaftsweise. Arch. Lebensmittelhyg., 32, 149-151.

Gravert H.O., Pabst K., Ordolff D., Treitel U., 1989. Milcherzeugung im alternativen landbau. K. M̈ilchw. Forsch., 41, 211-223.

Guinot-Thomas P., Jondreville C., Laurent F., 1991. Comparison of milk from farms with biological, conventional and transitional feeding. Milchwissenschaft, 46, 779-782.
Hansson I., Hamilton C., Ekman T., Forslund K., 2000. Carcass quality in certified organic production compared with conventional livestock production. J. Vet. Med., 47, 111-120.

Honikel K.O., 1998. Quality of ecologically produced foods of animal origin. Deutsch. Tierärztl. Wschr., 105, 327-329.

Hornick S.B., 1992. Factors affecting the nutritional quality of crops. Amer. J. Alternative Agr., 7, 63-69.

IFST, 2001. Organic food. Site internet de l'Institute of Food Science and Technology (UK) : http://www.ifst.org

INC, 1998. Manger bio: des malentendus à dissiper. 60 millions de consommateurs. (Avril 1999).

INC, 2001. La perception de la qualité alimentaire par les consommateurs. Etude réalisée en février 2001 par le Crédoc pour l'Institut National de la Consommation avec le soutien de la Communauté Européenne. http://www.inc60.fr/enquete_credoc/qualite.htm

Jensen T.K., Giwercman A., Carlsen E., Scheike T. Skakkebaek N.E., 1996. Semen quality among members of organic food associations in Zealand, Denmark. The Lancet, 347, 1844

Juhler R.K., Larsen S.B., Meyer O., Jensen N.D., Spano M., Giwercman A., Bonde J.P., 1999. Human semen quality in relation to dietary pesticide exposure and organic diet. Arch. Environ. Contam. Toxicol., 37, 415-423.

Jukes T.H., 1977. Organic food. CRC Crit. Rev. In Food Sci. Nutr., 9, 395-418.

Knöppler von H.O., Averdunk G., 1986. Vergleichende qualitätsuntersuchungen von konventionell und alternativ erzeugter kuhmilch. Arch. Lebensmittelhyg., 37, 94-96.

Larsen S.B., Spano M., Giwercman A., Bonde J.P., the ASCLEPIOS study group, 1999. Semen quality and sex hormones among organic and traditional Danish farmers. Occup. Environ. Med., 56, 139-144. 
Lecerf J.M., 1995. L'agriculture biologique. Intérêt en nutrition humaine? Cah. Nutr. Diét., 30, 349-357.

Lund P., 1991. Characterization of alternatively produced milk. Milchwissenschaft, 46, 166-169.

Maruejouls B., Goulard F., 1999. Résidus de pesticides dans le lait. Des résultats encourageants pour les produits de l'agriculture biologique. Alter Agri, 37, 10-13.

Marx H., Gedek B., Kollarczik B., 1995. Comparative investigations on mycotoxicological status of alternatively and conventionally grown crops. Z Lebensm. Unters. Forsch., 201, 83-86.

Oliva A., Spira A., Multigner L., 2001. Contribution of environmental factors to the risk of male infertility. Hum. Reprod., 16, 1768-1776.

Permin A., Bisgaard M., Frandsen F., Pearman M., Kold J., Nansen P., 1999. Prevalence of gastrointestinal helminths in different poultry production systems. Br. Poult Sci., 40, 439-443.

Plochberger K., 1989. Feeding experiments. A criterion for quality estimation of biologically and conventionally produced foods. Agric. Ecosyst. Environ., 27, 419-428

Plochberger K., Velimirov A., 1992. Are food preference tests with laboratory rats a proper method for evaluating nutritional quality? Biol. Agri. Horti., 8, 221-233.

Ratcliffe J.M., Schrader S.M., Steenland K., Clapp D.E., Turner T., Hornung R.W., 1987. Semen quality in papaya workers with long term exposure to ethylene dibromide. Br. J. Ind. Med., 44, 317-326.

Saffron L., 1999. Organic food and cancer risk. http://www.positivehealth.com/permit/Articles/Organic\%20 and\%20Vegetarian/safron30.htm

Skaug M.A., 1999. Analysis of Norwegian milk and infant formulas for ochratoxin A. Food Addit. Contam.,16, 75-78.
Sloan A.E. 1998. Organics: grown by the book. Food Technol., 52, 32

Smith B.L 1993. Organic food vs supermarket foods: element levels. J. Applied Nutr., 45, 35-39

Sundrum A., 2001. Organic livestock farming: a critical review. Livest. Prod. Sci., 67, 207-215.

Sundrum A., Bütfering L., Henning M., Hoppenbrock K.H., 2000. Effects of on-fram diets for organic pig production on performance and carcass quality. J. Anim. Sci., 78, $1199-1205$

Svensson C., Hessle A., Höglund J., 2000. Parasite control methods in organic and conventional dairy herds in Sweden. Livest. Prod. Sci., 66, 57-69.

Sylvander B., 1999. Les tendances de la consommation des produits biologiques en Europe: conséquences sur les perspectives d'évolution du secteur. ISARA-Université de Laval: organic agriculture faces it's development ; the futuLaval: organic agriculture face issues, Lyon, 6-8/12/1999.
re

Thamsborg S.M., Roepstorff A., Larsen M., 1999 Integrated and biological control of parasites in organic and conventional production systems. Vet. Parasitol., 84, 169-186.

Who, 1997. Conclusions of the joint FAO/IAEA/WHO study group on high dose irradiation. Weekly epidemiological record, 16 january 1998

Woese K., Lange D., Boess C., Bögl K.W., 1997. A comparison of organically and conventionally grown foods Results of a review of the relevant literature. J. Sci. Food Agric., 74, 281-293.

Woodward B.W., Fernandez M.I., 1999. Comparison of Woodward B.W., Fernandez M.I., 1999. Comparison of Carcass characteristics. Livest. Prod. Sci., 61, 225-231.

Worthington V., 1998. Effect of agricultural methods on nutritional quality: a comparison of organic with conventional crops. Altern. Ther. Health Med., 4, 58

\begin{abstract}

\section{Quality of organic animal products.}

'Organic' is a labelling term that denotes products that have been produced in accordance with organic standards. There is a community regulation on organic livestock production (council regulation $n^{\circ} 1804 / 1999$ ) that is applied in France (24/08/2000, CC REPAB F). There is a high demand for organic products in industrialised countries. It is therefore important to carefully consider the nutritional and sensory qualities and the safety of organic foods in order to identify problems and issues that require attention. The produc-

trends have been established in terms of nutritional and organoleptic quality differences between organic and conventional foods. Organically produced food has lower levels of veterinary drugs and pesticides. The 'organic' label provides also assurance to consumers that no food ingredient has been subject to irradiation and that genetically modified organisms have been excluded. However, it seems that organic farming could lead to a higher risk for the contamination of products by microbes present in animals and in manure. In summary, in some cases, organic food gets better marks, in others, conventionally produced food scores higher.
\end{abstract} tion of organic food of animal origin is done in many ways and uses many breeds. Therefore, a real comparison with conventionally produced food is difficult and there is a limited amount of published data. No clear
KOUBA M., 2002. Qualité des produits biologiques d'origine animale. INRA Prod. Anim., 15, 161-169. 
